\title{
Characterization by forbidden induced subgraphs of some subclasses of chordal graphs $^{0}$
}

\author{
Sérgio H. Nogueira ${ }^{1,2}$ \\ IEF , PPGMMC \\ Universidade Federal de Viçosa, Centro Federal de Educação Tecnológica de \\ Minas Gerais \\ Belo Horizonte, Brazil \\ Vinicius F. dos Santos ${ }^{1,3}$ \\ $D C C, P P G M M C$ \\ Universidade Federal de Minas Gerais, Centro Federal de Educação Tecnológica de \\ Minas Gerais \\ Belo Horizonte, Brazil
}

\begin{abstract}
Chordal graphs are the graphs in which every cycle of length at least four has a chord. A set $S$ is a vertex separator for vertices $a$ and $b$ if the removal of $S$ of the graph separates $a$ and $b$ into distinct connected components. A graph $G$ is chordal if and only if every minimal vertex separator is a clique. We study subclasses of chordal graphs defined by restrictions imposed on the intersections of its minimal separator cliques. Our goal is to characterize them by forbidden induced subgraphs. Some of these classes have already been studied such as chordal graphs in which two minimal separators have no empty intersection if and only if they are equal. Those graphs are known as strictly chordal graphs and they were first introduced as block
\end{abstract}


duplicate graphs by Golumbic and Peled [5], they were also considered in [7] and [2], showing that strictly chordal graphs are exactly the (gem, dart)-free graphs. ${ }^{0}$

Keywords: chordal graphs, forbidden induced subgraphs, minimal vertex separators.

\section{Introduction}

Throughout this text, a graph is always simple, finite and undirected. For sets $R$ and $S$, we denote $R \subseteq S$ if $R$ is a subset of $S$, and $R \subset S$ if $R$ is a proper subset of $S$. For a graph $G$ we denote the set of vertices by $V(G)$ and the set of edges by $E(G)$. Given two non-adjacent vertices $u$ and $v$ in the same connected component of $G$, a uv-separator is a set $S$ contained in $V(G)$ such that $u$ and $v$ are in different connected components of $G \backslash S$. This separator $S$ is minimal if no proper subset of $S$ is also a uv-separator. We will just say minimal vertex separator to refer to a set $S$ that is a $u v$-minimal separator for some pair of non-adjacent vertices $u$ and $v$ in $G$. A graph is chordal if it has no cycle of length at least four as induced graph. A clique is a maximal set of pairwise adjacent vertices. A clique tree of a connected chordal graph is any tree $\mathcal{T}$ whose vertices are the cliques of $G$ such that for every two cliques $C_{1}, C_{2}$ each clique on the path from $C_{1}$ to $C_{2}$ in $\mathcal{T}$ contains $C_{1} \cap C_{2}$.

In this paper we study the relationship between the family of minimal vertex separators and the structure of the graph. In particular we characterize the graph classes arising from properties imposed on the family of minimal vertex separators.

An important characterization of chordal graphs is due to Dirac:

Theorem 1.1 ([3]) A graph $G$ is chordal if and only if every minimal vertex separator of $G$ is a clique.

Two cliques $C_{1}, C_{2}$ of $\mathrm{G}$ form a separating pair if $C_{1} \cap C_{2}$ is non-empty, and every path in $\mathrm{G}$ from a vertex of $C_{1} \backslash C_{2}$ to a vertex of $C_{2} \backslash C_{1}$ contains a vertex of $C_{1} \cap C_{2}$. Every minimal separator of a chordal graph $\mathrm{G}$ is a clique and moreover, it is precisely the intersection of two cliques, as follows.

\footnotetext{
${ }_{0}$ Accepted for presentation at EURO/ALIO 2018

1 Partially supported by CAPES, CNPq and Fapemig

2 Email: sergiohnog@gmail.com

3 Email: viniciussantos@dcc.ufmg.br
} 
Theorem 1.2 ([6]) A set $S$ is a minimal separator of a chordal graph $G$ if and only if there exist maximal cliques $C_{1}, C_{2}$ forming a separating pair such that $S=C_{1} \cap C_{2}$.

A chordal graph can also be characterized using clique trees as follows:

Theorem 1.3 ([4]) A graph is chordal if and only if it has a clique tree.

Let $G$ be a chordal graph and let $\mathcal{T}(G)$ be a clique tree of $G$. The edges of $\mathcal{T}(G)$ can be labeled as the intersection of the endpoints, and these labels are exactly the minimal vertex separators. We denote by $\mathbf{S}_{\mathcal{T}}(G)$ the multiset of labels of the edges of $\mathcal{T}(G)$. A graph can have several distinct clique trees and when we are studying chordal graphs, we have the next result giving us that the multiset $\mathbf{S}_{\mathcal{T}}(G)$ is independent of the clique tree.

Theorem 1.4 ([1]) Let $G$ be a chordal graph. The multiset $\mathbf{S}_{\mathcal{T}}(G)$ of minimal vertex separators of $G$ is the same for every clique tree $\mathcal{T}(G)$.

In the light of Theorem 1.4 from now on we omit the subscript and use simply $\mathbf{S}(G)$.

A graph class $\mathcal{G}$ is hereditary if, for every $G \in \mathcal{G}$ and every induced subgraph $H$ of $G, H \in \mathcal{G}$.

Our goal is to characterize hereditary subclasses of chordal graphs by the intersection and containment relations of their minimal vertex separators and by forbidden induced subgraphs.

As we shall see, not every restriction on the minimal vertex separators leads to a hereditary graph class. In order to obtain characterization by forbidden induced subgraphs we will impose the additional requirement of being hereditary.

\section{Results}

We start with an auxiliary result showing that minimal vertex separators are, in some sense, hereditary.

Lemma 2.1 Let $G$ be a chordal graph, $S$ be a minimal vertex separator of $G$, $R$ be a proper subset of $S$ and $S^{\prime}=S \backslash R$. Then there exist cliques $C_{1}^{\prime}, C_{2}^{\prime}$ in $G \backslash R$ such that $S^{\prime}$ separates $C_{1}^{\prime}$ and $C_{2}^{\prime}$.

Proof. Since $S$ is a minimal vertex separator of $G$, there exist cliques $C_{1}, C_{2}$ in $G$ such that $S=C_{1} \cap C_{2}$. Let $C_{1}^{\prime}, C_{2}^{\prime}$ be cliques in $G^{\prime}=G \backslash R$ such that $\left(C_{i} \backslash R\right) \subset C_{i}^{\prime}, i=1,2$. $S^{\prime}$ separates $C_{1}^{\prime}$ and $C_{2}^{\prime}$, because if there exists a path 
in $G^{\prime}$ between a vertex $u_{1} \in C_{1}^{\prime}$ and $u_{2} \in C_{2}^{\prime}$ in $G^{\prime}$, then there exists a path from a vertex of $C_{1}$ to a vertex of $C_{2}$ in $G \backslash S$, contradicting the fact that $S$ separates $C_{1}$ and $C_{2}$ in $G$. Now suppose that $S^{\prime}$ is not minimal and let $R^{\prime}$ be a non-empty subset of $S^{\prime}$ such that $S^{\prime} \backslash R^{\prime}$ separates $C_{1}^{\prime}, C_{2}^{\prime}$. Then $S \backslash R^{\prime}$ separates $C_{1}, C_{2}$, contradicting the fact that $S$ is minimal vertex separator of $G$.

In the following, we will consider the graphs depicted in Figure 1:

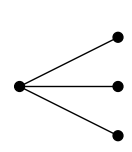

claw

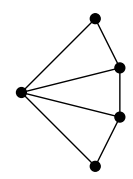

gem

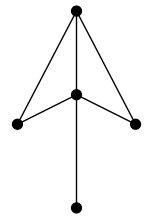

dart

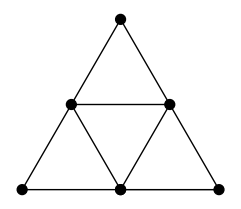

Hajós

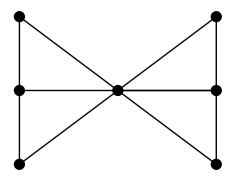

butterfly

Figure 1 - Forbidden Induced subgraphs considered in this work

Lemma 2.2 Let $G$ be a chordal graph. The following statements are equivalent:

- i) For every induced subgraph $G^{\prime}$ of $G$ and for every pair $S_{i}, S_{j} \in \mathbf{S}\left(G^{\prime}\right)$, $S_{i} \cap S_{j} \neq \emptyset$;

- ii) $G$ is $\left(P_{4}, 2 P_{3}\right)$-free.

Proof. Let $G$ be a graph satisfying $i$ ) and suppose that $i i$ ) is not valid. Suppose that there exists an induced subgraph $G^{\prime}$ of $G$ isomorphic to $P_{4}$ with vertices $v_{1}, v_{2}, v_{3}, v_{4}$ and cliques $C_{1}=\left\{v_{1}, v_{2}\right\}, C_{2}=\left\{v_{2}, v_{3}\right\}$ and $C_{3}=\left\{v_{3}, v_{4}\right\}$. Then we have $S_{1}=C_{1} \cap C_{2}=\left\{v_{2}\right\}, S_{2}=C_{2} \cap C_{3}=\left\{v_{3}\right\}$ and $S_{1} \cap S_{2}=\emptyset$, a contradiction. Now suppose we have an induced subgraph $G^{\prime}$ of $G$ isomorphic to $2 P_{3}$ with vertices $v_{1} v_{2} v_{3}$ and $v_{4} v_{5} v_{6}$ and cliques $C_{1}=\left\{v_{1}, v_{2}\right\}, C_{2}=\left\{v_{2}, v_{3}\right\}$, $C_{3}=\left\{v_{4}, v_{5}\right\}$ and $C_{4}=\left\{v_{5}, v_{6}\right\}$. Then we have $S_{1}=C_{1} \cap C_{2}=\left\{v_{2}\right\}$, $S_{2}=C_{3} \cap C_{4}=\left\{v_{5}\right\}$ and $S_{1} \cap S_{2}=\emptyset$, a contradiction. Hence $G$ is $\left(P_{4}, 2 P_{3}\right)$ free. Therefore $i) \Rightarrow i i)$.

Conversely, let $G$ be a graph satisfying $i i)$ and suppose that $i$ ) is not valid. Let $G^{\prime}$ be an induced subgraph of $G$. Let $\mathcal{T}^{\prime}$ be a clique tree of $G^{\prime}$ and $\mathbf{S}\left(G^{\prime}\right)$ be the multiset of minimal vertex separators of $G^{\prime}$. Suppose that there exist $S_{1}, S_{2} \in \mathbf{S}\left(G^{\prime}\right)$, with $S_{1} \cap S_{2}=\emptyset$. First, suppose that there exist adjacent edges $e_{1}, e_{2} \in E\left(\mathcal{T}^{\prime}\right)$ with labels $S_{1}, S_{2}$ and let $C_{1}, C_{2}, C_{3}$ be cliques such that $S_{1}=C_{1} \cap C_{2}$ and $S_{2}=C_{2} \cap C_{3}$. Suppose $x \in S_{1}$ and $y \in S_{2}$. Since the cliques are maximal there must exist $a \in C_{1} \backslash C_{2}$ and $b \in C_{3} \backslash C_{2}$. Then $\{a, x, y, b\}$ induces a $P_{4}$. Now suppose that there exist non-adjacent edges $e_{1}, e_{2} \in E\left(\mathcal{T}^{\prime}\right)$ with labels $S_{1}, S_{2}$ and let $C_{1}, C_{2}, C_{3}, C_{4}$ be cliques such that $S_{1}=C_{1} \cap C_{2}$ and 
$S_{2}=C_{3} \cap C_{4}$. Without loss of generality we can consider that the path in $\mathcal{T}^{\prime}$ from $\left\{C_{1}, C_{2}\right\}$ to $\left\{C_{3}, C_{4}\right\}$ contains $C_{2}$ and $C_{3}$. Suppose $x \in S_{1}$ and $y \in S_{2}$. Since the cliques are maximal we must have $a \in C_{1} \backslash C_{2}, b \in C_{4} \backslash C_{3}$ and $c \in$ $C_{2} \backslash C_{1}$. If $x y \in E\left(G^{\prime}\right)$ then $\{a, x, y, b\}$ is an induced $P_{4}$ of $G$; otherwise if $c y \in E\left(G^{\prime}\right)$ then $\{a, x, c, y\}$ induces a $P_{4}$; else there exists $d \in C_{3} \backslash\{a, b, c, x, y\}$. If $d x \in E\left(G^{\prime}\right)$ then $\{x, d, y, b\}$ is $P_{4}$; if $c d \in E\left(G^{\prime}\right)$ then $\{x, c, d, y\}$ is $P_{4}$ else $\{a, x, c\},\{d, y, b\}$ induce $2 P_{3}$, a contradiction. Therefore $\left.i i\right) \Rightarrow i$ ).

Lemma 2.3 Let $G$ be a chordal graph. The following statements are equivalent:

- i) For every $G^{\prime}$ induced subgraph of $G$ and for every pair $S_{i}, S_{j} \in \mathbf{S}\left(G^{\prime}\right)$, $S_{i} \neq S_{j}$.

- ii) $G$ is claw-free.

Proof. Let $G$ be a graph satisfying $i$ ) and suppose that $i i$ ) is not valid. Let $G^{\prime}$ be an induced subgraph of $G$ and let $\{x\}\{a, b, c\}$ be an induced claw of $G^{\prime}$, with cliques $C_{1}=\{x, a\}, C_{2}=\{x, b\}, C_{3}=\{x, c\}$. Then we have $S_{1}=$ $C_{1} \cap C_{2}=\{x\}=C_{2} \cap C_{3}=S_{2}$, a contradiction. Hence $G$ is claw-free and i) $\Rightarrow i i)$.

Conversely let $G$ be a graph satisfying $i i)$ and suppose that $i$ ) is not valid. Let $G^{\prime}$ be an induced subgraph of $G$. Let $\mathcal{T}^{\prime}$ be a clique tree of $G^{\prime}, \mathbf{S}\left(G^{\prime}\right)$ be the multiset of minimal vertex separators of $G^{\prime}$ and suppose that $\exists S_{1}, S_{2} \in \mathbf{S}\left(G^{\prime}\right)$, with $S_{1}=S_{2}$. First, suppose that there exist adjacent edges $e_{1}, e_{2} \in E\left(\mathcal{T}^{\prime}\right)$ with labels $S_{1}, S_{2}$ and let $C_{1}, C_{2}, C_{3}$ be cliques such that $S_{1}=C_{1} \cap C_{2}$ and $S_{2}=C_{2} \cap C_{3}$. Since the separators are equal, take $x \in S_{1} \cap S_{2}$. Since the cliques are maximal there must exist $a \in C_{1} \backslash C_{2}, b \in C_{3} \backslash C_{2}$ and $c \in C_{2} \backslash\left(C_{1} \cup C_{3}\right)$. Then $\{x, a, b, c\}$ induces a claw, a contradiction. Now suppose that $\nexists e_{1}, e_{2} \in$ $E\left(\mathcal{T}^{\prime}\right)$ adjacent with labels $S_{1}, S_{2}$ and let $C_{1}, C_{2}, C_{3}, C_{4}$ be distinct cliques such that $S_{1}=C_{1} \cap C_{2}$ and $S_{2}=C_{3} \cap C_{4}$. Without loss of generality we can consider that every path in $\mathcal{T}^{\prime}$ from $\left\{C_{1}, C_{2}\right\}$ to $\left\{C_{3}, C_{4}\right\}$ contains $C_{2}$ and $C_{3}$. Let $x \in \bigcap_{i=1, \ldots, 4} C_{i}$. Since the cliques are maximal we must have $a \in C_{1} \backslash C_{2}$, $b \in C_{4} \backslash C_{3}$ and $c \in C_{2} \backslash C_{1}$ and then $\{x, a, b, c\}$ induces a claw, a contradiction. Hence $i i) \Rightarrow i$ ).

Due to space restrictions the proofs of the following two lemmas will be omitted.

Lemma 2.4 Let $G$ be a chordal graph. The following statements are equivalent:

- i) For every $G^{\prime}$ induced subgraph of $G$ and for every pair $S_{i}, S_{j} \in \mathbf{S}\left(G^{\prime}\right), S_{i}$ 
is not strictly contained in $S_{j}$;

- ii) $G$ is dart-free.

Lemma 2.5 Let $G$ be a chordal graph. The following statements are equivalent:

- i) For every $G^{\prime}$ induced subgraph of $G$ and for every pair $S_{i}, S_{j} \in \mathbf{S}\left(G^{\prime}\right)$, $S_{i} \subseteq S_{j}$ or $S_{j} \subseteq S_{i}$;

- ii) $G$ is (gem, butterfly)-free.

Let $\mathcal{H}$ be a hereditary subclass of chordal graphs. Let $G \in \mathcal{H}, \mathcal{T}$ be a clique tree of $G$ and $\mathbf{S}(G)$ be the multiset of minimal vertex separators of $G$. For each pair $S_{i}, S_{j} \in \mathbf{S}(G)$ one of the following situations holds:

- (a) Disjunction: $S_{i} \cap S_{j}=\emptyset$.

- (b) Equality: $S_{i}=S_{j}$.

- (c) Containment: $S_{i} \subset S_{j}$ or $S_{j} \subset S_{i}$.

- (d) Overlap: $S_{i} \nsubseteq S_{j}$ and $S_{j} \nsubseteq S_{i}$.

Remark 2.6 Since we are interested in a hereditary class $\mathcal{H}$, we can note that, by Lemma 2.1, if we have Containment then we must allow Equality and if we have Overlap then we must allow Equality and Disjunction.

Remark 2.7 And again by hereditarity we can note that if a class is claw-free then it is dart-free; if it is $P_{4}$-free then it is gem-free and if it is dart-free or $2 P_{3}$-free then it is butterfly-free.

Hence all possible combinations of properties $(a)-(d)$ are characterized in the following theorem.

Theorem 2.8 Let $G$ be a chordal graph. Then for every $G^{\prime}$ induced subgraph of $G$ and for every pair $S_{i}, S_{j} \in \mathbf{S}\left(G^{\prime}\right), i \neq j$, we have:

- (i) $S_{i} \cap S_{j}=\emptyset \Leftrightarrow G$ is (claw, gem)-free.

- (ii) $S_{i}=S_{j} \Leftrightarrow G$ is (P4, gem, butterfly)-free.

- (iii) $S_{i} \cap S_{j}=\emptyset$ or $S_{i}=S_{j} \Leftrightarrow G$ is (dart, gem)-free.

- (iv) $S_{i} \cap S_{j}=\emptyset$ or $S_{i}=S_{j}$ or $S_{i} \subset S_{j}$ or $S_{j} \subset S_{i} \Leftrightarrow G$ is (gem, butterfly)free.

- (v) $S_{i} \cap S_{j}=\emptyset$ or $S_{i}=S_{j}$ or $\left(S_{i} \nsubseteq S_{j}\right.$ and $\left.S_{j} \nsubseteq S_{i}\right) \Leftrightarrow G$ is dart-free.

- (vi) $S_{i}=S_{j}$ or $S_{i} \subset S_{j}$ or $S_{j} \subset S_{i} \Leftrightarrow G$ is $\left(2 P_{3}, P_{4}\right)$-free.

Proof. It follows from Lemmas 2.2-2.5 and Remark 2.7. 
We remark that (iii) had been previously proved in [7] and [2].

We now move our attention to Helly Property. Let $\mathcal{F}$ be a family of subsets of a set $\mathcal{S}$. We say that $\mathcal{F}$ satisfies the Helly property when every subfamily $\mathcal{F}^{\prime}$ of $\mathcal{F}$ consisting of pairwise intersecting subsets satisfies $\cap_{F \in \mathcal{F}^{\prime}} F \neq \emptyset$.

We say that a set $X$ is a witness that the Helly property does not hold if:

- $S_{i} \cap S_{j} \neq \emptyset, \forall S_{i}, S_{j} \in X$ and

- $\bigcap_{S_{i} \in X} S_{i}=\emptyset$.

Lemma 2.9 Let $G$ be a chordal graph such that $\mathbf{S}(G)$ does not satisfy the Helly property and such that for every induced subgraph $G^{\prime}$ the family $\mathbf{S}\left(G^{\prime}\right)$ satisfies the Helly property. Let $\mathcal{T}$ be a clique tree of $G$ and $\mathbf{S}_{\ell}(T)$ be the set of minimal vertex separators incident to leaves of $\mathcal{T}$. Then $\mathbf{S}_{\ell}(T)$ is a witness.

Proof. If there exist separators $S_{i}, S_{j} \in \mathbf{S}_{\ell}(T)$ with $S_{i} \cap S_{j}=\emptyset$ then $S_{i}, S_{j}$ cannot be simultaneously in a witness. But then there exists witness $R \subset$ $\mathbf{S}(G) \backslash S_{i}$ or $R \subset \mathbf{S}(G) \backslash S_{j}$, wich contradicts the minimality of $G$. Then suppose that $S_{i} \cap S_{j} \neq \emptyset$ for all pairs $S_{i}, S_{j} \in \mathbf{S}_{\ell}(T)$. Since $\mathbf{S}_{\ell}(T)$ is not a witness there exists $x \in \bigcap_{S_{i} \in \mathbf{S}_{\ell}(T)} S_{i}$ and then $x$ is universal in $G$ and this implies that $x$ belongs to all minimal vertex separators of $G$. But this implies that $\mathbf{S}(G)$ satisfies the Helly property, a contradiction.

Theorem 2.10 Let $\mathcal{G}$ be the hereditary class of chordal graphs such that $\forall G \in$ $\mathcal{G}, \mathbf{S}(G)$ satisfies the Helly property. Then for any chordal graph $G, G \in \mathcal{G} \Leftrightarrow$ $G$ is Hajós-free.

Proof. Let $G \in \mathcal{G}$ and let $x y z a b c$ be an induced Hajós of $G$, with cliques $C_{1}=\{x, y, a\}, C_{2}=\{x, z, b\}, C_{3}=\{y, z, c\}, C_{4}=\{x, y, z\}$. Let $S_{1}=C_{1} \cap$ $C_{4}=\{x, y\}, S_{2}=C_{2} \cap C_{4}=\{x, z\}, S_{3}=C_{3} \cap C_{4}=\{y, z\}$. Then we have $S_{1} \cap S_{2}=\{x\}, S_{1} \cap S_{3}=\{y\}, S_{2} \cap S_{3}=\{z\}$ and $S_{1} \cap S_{2} \cap S_{3}=\emptyset$, so $\mathbf{S}(G)$ does not satisfy the Helly property.

Conversely let $G \notin \mathcal{G}$ and let $G^{\prime}$ be an induced subgraph of $G$ minimal in relation to the property that $\mathbf{S}\left(G^{\prime}\right)$ does not satisfy the Helly property and let $\mathcal{T}^{\prime}$ be a clique tree of $G^{\prime}$. By the previous Lemma, $\mathbf{S}_{\ell}\left(T^{\prime}\right)$ is a witness. Let $S_{1}, S_{2} \in \mathbf{S}_{\ell}\left(T^{\prime}\right)$. Then $\exists x_{1} \in S_{1} \cap S_{2}$ and $\exists S_{i} \in \mathbf{S}_{\ell}\left(T^{\prime}\right)$ such that $x_{1} \notin S_{i}$. Without lost of generality, let $S_{i}=S_{3}$. Note that if $A, B, C \in \mathbf{S}_{\ell}\left(T^{\prime}\right)$ then $A \cap B \nsubseteq C$. Indeed, suppose $A \cap B \subseteq C$. Since $\mathbf{S}_{\ell}\left(T^{\prime}\right)$ is a witness, we know that $\bigcap_{X \in \mathbf{S}_{\ell}\left(T^{\prime}\right)} X=\emptyset$. Then we have:

$$
\left(\bigcap_{X \in \mathbf{S}_{\ell}\left(T^{\prime}\right) \backslash\{A, B, C\}} X\right) \cap A \cap B \cap C=\emptyset \Rightarrow
$$




$$
\begin{gathered}
\left(\bigcap_{X \in \mathbf{S}_{\ell}\left(T^{\prime}\right) \backslash\{A, B, C\}} X\right) \cap A \cap B=\emptyset \Rightarrow \\
\bigcap_{X \in \mathbf{S}_{\ell}\left(T^{\prime}\right) \backslash\{C\}} X=\emptyset .
\end{gathered}
$$

But this implies that there exists a proper subset of $\mathbf{S}_{\ell}\left(T^{\prime}\right)$ that does not satisfy the Helly property, contradicting the minimality of $G^{\prime}$. Then we can suppose that $\exists y \in S_{3} \cap S_{1} \backslash S_{2}$ and $\exists z \in S_{3} \cap S_{2} \backslash S_{1}$. Let $C_{1}, C_{2}, C_{3}$ be the leaves of edges labeled by $S_{1}, S_{2}, S_{3}$ respectively in $\mathcal{T}^{\prime}$. Let $v_{i}$ be an exclusive vertex of $C_{i}, i=1 \ldots 3$. Then $\left\{x, y, z, v_{1}, v_{2}, v_{3}\right\}$ induces a Hajós.

\section{References}

[1] Blair, J.R.S. and Peyton, B., An Introduction to Chordal Graphs and Clique Trees, Graph Theory and Sparc Matrix Computation, IMA 56 (1992) 1-29.

[2] De Caria, P. and Gutiérrez, M., On basic chordal graphs and some of its subclasses, Discrete Applied Mathematics, 210 (2016) 261-276.

[3] Dirac, G. A., On rigid circuit graphs, Abh. Math. Sem. Univ. HamDurg, 25 (1961) 71-76.

[4] Gavril, F., The intersection graphs of subtrees in trees are exactly the chordal graphs, Journal of Combinatory Theory, B 116 (1974) 47-56.

[5] Golumbic, M. C. and Peled, U. N., Block duplicate graphs and a hierarchy of chordal graphs, Discrete Applied Mathematics, 124 (2002) 67-71.

[6] Habib, M. and Stacho, L., Reduced clique graphs of chordal graphs, European Journal of Combinatorics, 33 (2012) 712-735.

[7] Kennedy, W., "Strictly chordal graphs and phylogenetic roots", Master Thesis, University of Alberta, 2005. 